\title{
Magnetocaloric effect and magnetic properties of $\mathrm{Tb}_{0.9} \mathrm{Sn}_{0.1} \mathrm{MnO}_{3}$
}

\author{
F. Wolff Fabris ${ }^{\text {a) }}$ \\ SUPRATECS, Montefiore Institute of Electricity, B28, University of Liège, B-4000, Liège, Belgium
}

M. Pekala

Department of Chemistry, Warsaw University, Al. Zwirki i Wigury 101, 02-089 Warsaw, Poland

V. Drozd

Department of Chemistry, National Taiwan University, Roosveld Rd., Section 4, Taipei, Taiwan

J-F. Fagnard and Ph. Vanderbemden

SUPRATECS, Montefiore Institute of Electricity, B28, University of Liège, B-4000, Liège, Belgium

Ru-Shi Liu

Department of Chemistry, National Taiwan University, Roosveld Rd., Section 4, Taipei, Taiwan

\author{
M. Ausloos \\ SUPRATECS, B5, University of Liège, B-4000, Liège, Belgium
}

(Received 22 November 2006; accepted 13 March 2007; published online 18 May 2007)

\begin{abstract}
The magnetocaloric effect in magnetic materials is of great interest nowadays. In this article we present an investigation about the magnetic properties near the magnetic transition in a polycrystalline sample of a manganite $\mathrm{Tb}_{0.9} \mathrm{Sn}_{0.1} \mathrm{MnO}_{3}$. Particularly, we are interested in describing the nature of the magnetic interactions and the magnetocaloric effect in this compound. The temperature dependence of the magnetization was measured to determine the characteristics of the magnetic transition and the magnetic entropy change was calculated from magnetization curves at different temperatures. The magnetic solid is paramagnetic at high temperatures. We observe a dominant antiferromagnetic interaction below $T_{n}=38 \mathrm{~K}$ for low applied magnetic fields; the presence of $\mathrm{Sn}$ doping in this compound decreases the Néel temperature of the pure $\mathrm{TbMnO}_{3}$ system. A drastic increase in the magnetization as a function of temperature near the magnetic transition suggests a strong magnetocaloric effect. We found a large magnetic entropy change $\Delta S_{M}(T)$ of about $-4 \mathrm{~J} / \mathrm{kg} \mathrm{K}$ at $H=3 \mathrm{~T}$. We believe that the magnetic entropy change is associated with the magnetic transition and we interpret it as due to the coupling between the magnetic field and the spin ordering. This relatively large value and broad temperature interval (about $35 \mathrm{~K}$ ) of the magnetocaloric effect make the present compound a promising candidate for magnetic refrigerators at low temperatures. (C) 2007 American Institute of Physics. [DOI: 10.1063/1.2732453]
\end{abstract}

\section{INTRODUCTION}

The magnetocaloric effect results in a material temperature change under an external magnetic field change. ${ }^{1}$ An applied field will tend to align the magnetic spins and thus decreases the total entropy of the spin system. Therefore the magnetocaloric effect is the result of the magnetic entropy change $\Delta S_{M}(T)$ arising from the coupling of the magnetic spin system of the solid with the external applied magnetic field. Recall that the total entropy of a magnetic solid is the sum of the lattice, electronic, and magnetic entropies. In most cases, the lattice and electronic entropies are essentially independent of the magnetic field $H$, whereas the magnetic entropy is strongly dependent on $H$. Therefore it is of interest to look for materials presenting such high changes, and understand the microscopic causes. It is of interest to search for useful coupling effects.

In fact magnetoelectrics and multiferroics materials, which exhibit mutual coupling of ferroelectricity and magnetism, have been the subject of great interest in recent years.

\footnotetext{
${ }^{\text {a) }}$ Present address: MST-NHMFL, Los Alamos National Laboratory, Los Alamos, New Mexico 87545, USA.
}

This is the case for $R \mathrm{MnO}_{3}(R=\mathrm{Sc}, \mathrm{Y}, \mathrm{Er}, \mathrm{Ho}, \mathrm{Tm}, \mathrm{Yb}, \mathrm{Lu}$, $\mathrm{Tb})$ rare-earth manganites; they form an interesting family of compounds showing a wide variety of physical properties. In the orthorhombically distorted $\mathrm{TbMnO}_{3}$ compound, being a reference for our analysis, ${ }^{2,3}$ the coexistence of antiferromagnetism and ferroelectricity is observed at low temperatures. This coexistence and the strong coupling of antiferromagnetism and ferroelectricity suggest the presence of a nonconventional coupling mechanism involving competing spin interactions. A multiferroic behavior arises as a consequence of the release of frustration of the applied magnetic field $H$. In this compound, ferroelectricity arises below the Néel temperature from a coupling to the lattice of an incommensurate modulation of the magnetic structure below $27 \mathrm{~K}_{\text {that }}{ }^{2-5}$ is caused from frustration in the ordering of the $\mathrm{Mn} d$ orbitals. In fact, an anomaly has been reported ${ }^{2}$ in pure $\mathrm{TbMnO}_{3}$ samples at $\sim 42 \mathrm{~K}$ which is attributed to a sine wave ordering of the $\mathrm{Mn}^{+3}$ moments.

Recent papers aim to study the doping effect substituting the rare-earth element in these manganite compounds ${ }^{6-9}$ and many interesting effects have been shown on the magnetic properties, particularly on the magnetocaloric effect (MCE). 


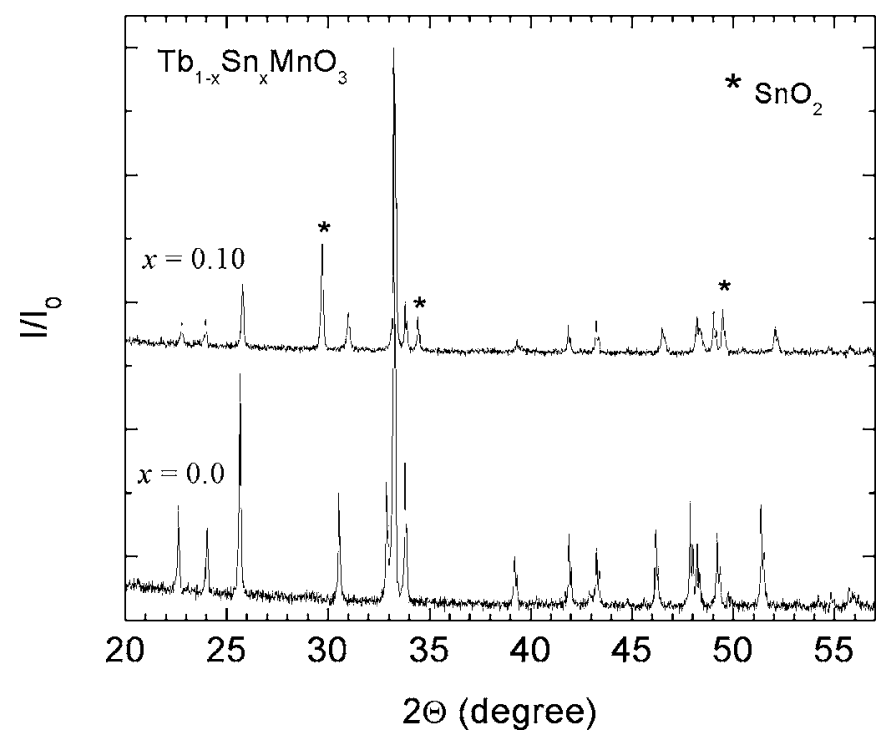

FIG. 1. X-ray diffraction patterns of our $\mathrm{Tb}_{1-x} \mathrm{Sn}_{x} \mathrm{MnO}_{3}$ samples with $x=0$ and 0.1 .

Moreover, the possibility of using magnetic materials, which exhibit this effect as a magnetic refrigerant, has given some fundamental right for the research on the magnetocaloric properties in such magnetic systems. One of the main goals of recent studies on MCE is thus to find useful magnetic materials which have a large entropy change at low magnetic fields.

In this article we present an experimental work on the magnetic properties in a polycrystalline sample of a rareearth doped manganite $\mathrm{Tb}_{0.9} \mathrm{Sn}_{0.1} \mathrm{MnO}_{3}$. Particularly, we are interested in studying the nature of the magnetic interactions and the magnetic entropy change $\Delta S_{M}(T)$ in this compound. It is of interest to find whether magnetic transitions exist and their effect on MCE.

\section{EXPERIMENT}

The polycrystalline samples $\mathrm{Tb}_{0.9} \mathrm{Sn}_{0.1} \mathrm{MnO}_{3}$ and $\mathrm{TbMnO}_{3}$ were prepared by conventional solid state reaction method starting from stoichiometric mixtures of the precursor powders $\mathrm{Tb}_{4} \mathrm{O}_{7}, \mathrm{MnO}_{2}$, and $\mathrm{SnO}_{2}$. These reagents were mixed in agate mortar and sintered at $1000-1400{ }^{\circ} \mathrm{C}$ with intermediate grinding after $24 \mathrm{~h}$ of sintering at 1000, 1100, 1200 , and $1300{ }^{\circ} \mathrm{C}$. The ac magnetic susceptibility (at $10 \mathrm{Oe}$ magnetic field amplitude), dc magnetization and magnetic isotherms were measured using a Quantum Design PPMS-7 magnetometer. We applied magnetic fields up to $3 \mathrm{~T}$ under the protocols zero field cooled (ZFC) and field cooled (FC) and the temperature range varies from $9 \mathrm{~K}$ up to room temperature.

Figure 1 shows $\mathrm{x}$-ray diffraction patterns of the $\mathrm{Tb}_{0.9} \mathrm{Sn}_{0.1} \mathrm{MnO}_{3}$ sample as compared to the undoped $\mathrm{TbMnO}_{3}$. The $\mathrm{SnO}_{2}$ peaks occur at angles $2 \theta=29.7^{\circ}, 34.4^{\circ}$, and $49.5^{\circ}$. It is clear that the Sn doping causes structural changes as soon as the peak intensities vary. The lattice parameters of the orthorhombic $\mathrm{Tb}_{0.9} \mathrm{Sn}_{0.1} \mathrm{MnO}_{3}$ sample are $a$ $=0.52990(7) \mathrm{nm}, \quad b=0.57661(7) \mathrm{nm}$, and $c=0.74273(8)$ $\mathrm{nm}$. The volume of the unit cell is $0.22694(5) \mathrm{nm}^{3}$. This

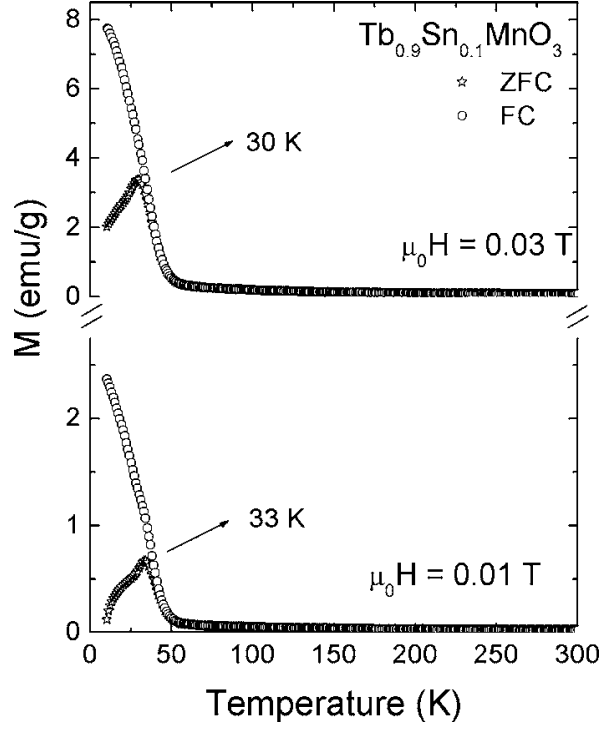

FIG. 2. Zero-field-cooled (ZFC) and field-cooled (FC) dc magnetization as a function of temperature measured at an applied magnetic field of 0.01 and $0.03 \mathrm{~T}$.

proves that a $10 \%$ content of Sn causes a lattice compression as the corresponding parameters for $\mathrm{TbMnO}_{3}$ at room temperature are as follows: $a=0.5293 \mathrm{~nm}, b=0.5838 \mathrm{~nm}, c$ $=0.7403 \mathrm{~nm}$ and the unit cell volume is $0.22876 \mathrm{~nm}^{3}$. It is worth noting that the lattice parameters for our $\mathrm{TbMnO}_{3}$ sample are very close to those reported by Cui et al. ${ }^{10}$ for the same system.

\section{LOW MAGNETIC FIELD PROPERTIES}

The temperature dependence for the dc magnetization at low applied magnetic fields is presented in Fig. 2. We observe a drastic change of the magnetization at the vicinity of the magnetic transition, which is indicative of a large magnetic entropy change. This figure also shows a divergence between the field-cooled (FC) and zero-field-cooled (ZFC) magnetization developing below the so-called irreversibility temperature. The irreversibility temperature decreases from 33 down to $30 \mathrm{~K}$ with the external magnetic field increasing from 0.01 to $0.03 \mathrm{~T}$. From this figure we can see that there is clearly a cusp in ZFC curves at the so-called freezing or spin-glass temperature $T_{g}$. The irreversibility phenomenon is a typical feature of spin-glass- or cluster-glass-like states, ${ }^{11}$ when a frustration between the antiferromagnetic and ferromagnetic ordering occurs. In a case of manganites these interactions are ascribed to the superexchange and double exchange interactions, respectively. ${ }^{12}$

Taking the derivative $(d M / d T)$ of the dc magnetization measured as a function of temperature in the case of $0.01 \mathrm{~T}$ (shown in Fig. 3) one may ascribe the minimum at $\mathbf{T}_{N}$ $=38 \mathrm{~K}$ to the Néel temperature of the sine-wave ordering of the $\mathrm{Mn}^{3+}$ magnetic moments. The Néel temperature is slightly reduced as compared with $41 \mathrm{~K}$ reported for the $\mathrm{Sn}$-free $\mathrm{TbMnO}_{3}$ system. ${ }^{3}$ The second minimum in our sample at $\mathbf{T}_{\text {loc }}=25 \mathrm{~K}$ corresponds to the transition observed for $\mathrm{TbMnO}_{3}$ at $27 \mathrm{~K}$ and at zero magnetic field ${ }^{3}$ likely re- 


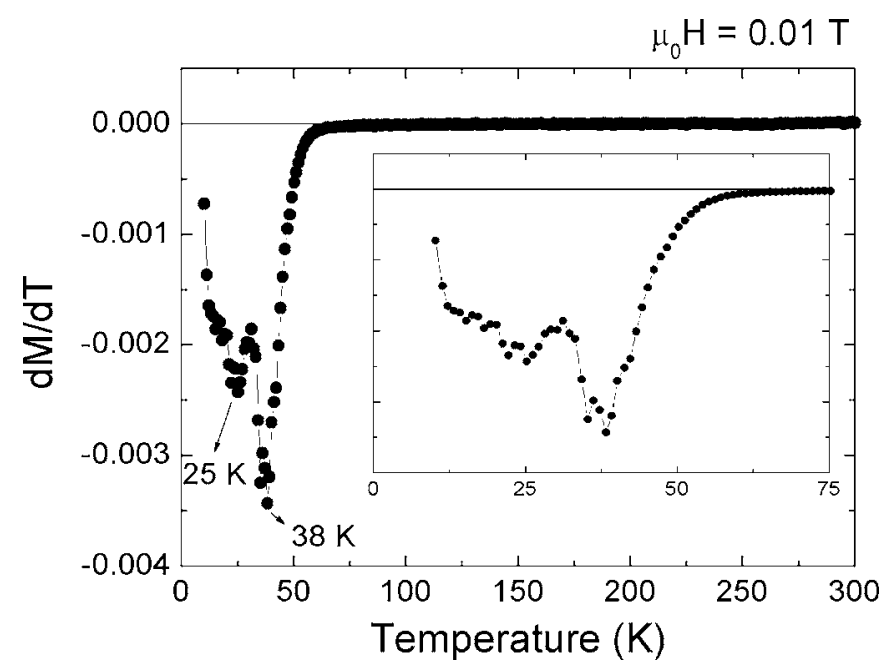

FIG. 3. Plot of $d M / d T$ in a function of temperature at a magnetic field of $0.01 \mathrm{~T}$; two minima are seen at low temperatures of 25 and $38 \mathrm{~K}$.

veals commensurate-incommensurate transition within the $\mathrm{Mn}^{3+}$ system. The magnetic moments become locked when applying the magnetic field of $0.03 \mathrm{~T}$.

We plot the inverse ac susceptibility curve as a function of temperature, the so-called Curie-Weiss plot, which is shown in Fig. 4. Using this plot and adjusting a corresponding Curie-Weiss fit given by the relationship $\chi=C /(T-\theta)$, we found a linear dependence above $70 \mathrm{~K}$, which extends up to higher temperatures. So, the magnetic behavior obeys a well-defined Curie-Weiss law up to room temperatures and the compound is in a typical paramagnetic state. Several fits were adjusted at this region by changing the temperature range for the fit. In all cases, the extrapolation of this linear regime shows a negative Weiss temperature $\theta$. The medium value for this temperature was found at $\theta=-37( \pm 2) \mathrm{K}$, indicating that the nature of the dominant magnetic interactions in this compound is antiferromagnetic. Previous reports of neutron-scattering measurements ${ }^{13-15}$ on $\mathrm{RE}^{-\mathrm{MnO}_{3}}$ compounds indicate that the $\mathrm{Mn}$ moments lie in an incommensurate antiferromagnetic phase.

\section{HIGH MAGNETIC FIELD PROPERTIES}

We have measured (Fig. 5) magnetic isotherms at temperatures from 9 to $67 \mathrm{~K}$. The temperature interval between the isotherms was $2 \mathrm{~K}$ and the magnetic field for each iso-

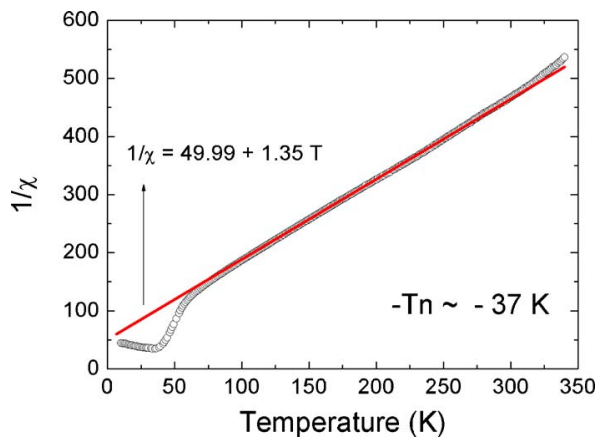

FIG. 4. (Color online) Inverse of the magnetic susceptibility as a function of temperature. The line corresponds to the Curie-Weiss fit.

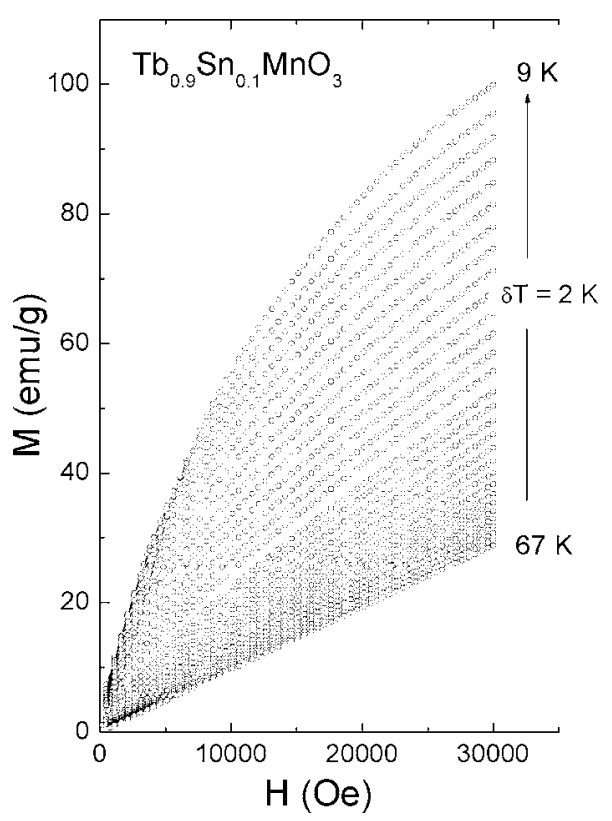

FIG. 5. Magnetic isotherms measured at different temperatures for our $\mathrm{Tb}_{0.9} \mathrm{Sn}_{0.1} \mathrm{MnO}_{3}$ sample.

therm was varied between zero and $3 \mathrm{~T}$, in steps of $0.05 \mathrm{~T}$. For this range of applied magnetic field the magnetic behavior of the sample is typically of a ferromagnetic system, however, the saturation magnetization is not fully reached at this maximum applied magnetic field. Thus one may conclude that in contrast to the antiferromagnetic behavior observed at low magnetic fields, the strong magnetic fields overcome the weak local magnetic anisotropy and lead to the ferromagnetic ordering.

The so-called Arrott plot $M^{2}$ vs $H / M$ in Fig. 6 exhibits the positive slope of the curves at all temperatures studied. This indicates that a transition between the magnetically ordered and the paramagnetic phases is of the second order. ${ }^{16}$ The Arrott plot did not allow one to determine a critical temperature of magnetic transition since the extensions of the plots do not reach a center of the $M^{2}$ and $H / M$ coordinate system. This suggests that at least due to a competition between the ferromagnetic and antiferromagnetic interac-

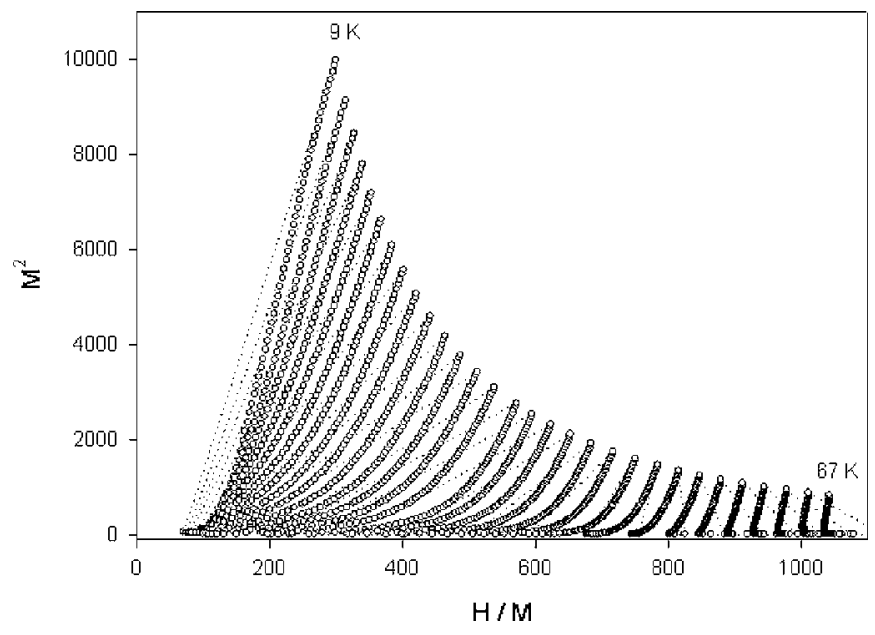

FIG. 6. Arrott plot $M^{2}$ vs $H / M$. 
tions, the magnetic transition is broadly spread both in temperature and in magnetic field. The broadening effect may be additionally enhanced by the local inhomogeneities of the sample.

\section{MAGNETOCALORIC EFFECT}

The isothermal magnetic entropy change $\Delta S_{M}(T)$, which is associated with the magnetocaloric effect, can be calculated from measurements of magnetization as a function of the applied magnetic field and temperature (indirect measurement technique of the magnetocaloric effect). According to the classical thermodynamics theory, the magnetic entropy change produced by varying the magnetic field from 0 to $H_{\max }$ is given by ${ }^{17}$

$$
\Delta S_{M}(T, H)=\int_{0}^{H_{\max }}\left(\frac{\partial S}{\partial H}\right)_{T} d H .
$$

By using Maxwell's thermodynamic relation

$$
\left(\frac{\partial S}{\partial H}\right)_{T}=\left(\frac{\partial M}{\partial T}\right)_{H},
$$

the magnetic entropy change can be rewritten as follows:

$$
\Delta S_{M}(T, H)=\int_{0}^{H_{\max }}\left(\frac{\partial M}{\partial T}\right)_{H} d H .
$$

The integral in Eq. (3) corresponds to the area enclosed between two isothermal magnetization curves $M(H, T)$ and $M(H, T+\delta T)$, where $\delta T$ is the temperature difference between two isotherms. We calculated this area using a precise numerical integration. A complete $\Delta S_{M}(T)$ versus temperature curve can be derived from a series of isothermal magnetization curves obtained at discrete temperatures with an appropriate interval (relatively small) of temperatures $\delta T$ and magnetic fields $\Delta H$.

The magnetic entropy change $-\Delta S_{M}(T)$ as a function of temperature plotted in Fig. 7 for various magnetic fields, has a characteristic shape. ${ }^{18}$ It is worth noting that the $-\Delta S_{M}(T)$ maximum is located near $25 \mathrm{~K}$-around the commensurateincommensurate transition of the $\mathrm{Mn}^{3+}$ system. The $-\Delta S_{M}(T)$ maximum magnitude increases with increasing magnetic field up to $-4 \mathrm{~J} / \mathrm{Kg} \mathrm{K}$ at $3 \mathrm{~T}$. The $-\Delta S_{M}(T)$ curve is rather broad and the region of $-\Delta S_{M}(T)$ maximum is enlarging in higher magnetic fields. One may see in Fig. 7 that the position of this broad maximum shifts from 25 to $23 \mathrm{~K}$ when the magnetic field reaches $3 \mathrm{~T}$. This shift is in contrast to the opposite tendency observed for, e.g., $\mathrm{La}_{0.7} \mathrm{Ca}_{0.3} \mathrm{MnO}_{3}$ manganites, ${ }^{19}$ when the Curie temperature is enhanced by a magnetic field. A more detailed inspection of the top part of the $-\Delta S_{M}(T)$ curve (especially at 2 and $3 \mathrm{~T}$ ) suggests that it may be composed of two overlapping components corresponding to the transitions at $T_{N}$ and $T_{\text {loc }}$. A similar double peak structure of the $-\Delta S_{M}(T)$ curve is reported in Ref. 20. To clarify whether the broad and smooth maximum corresponds to a two peak structure might require further work applying smaller temperature steps. In so doing one may

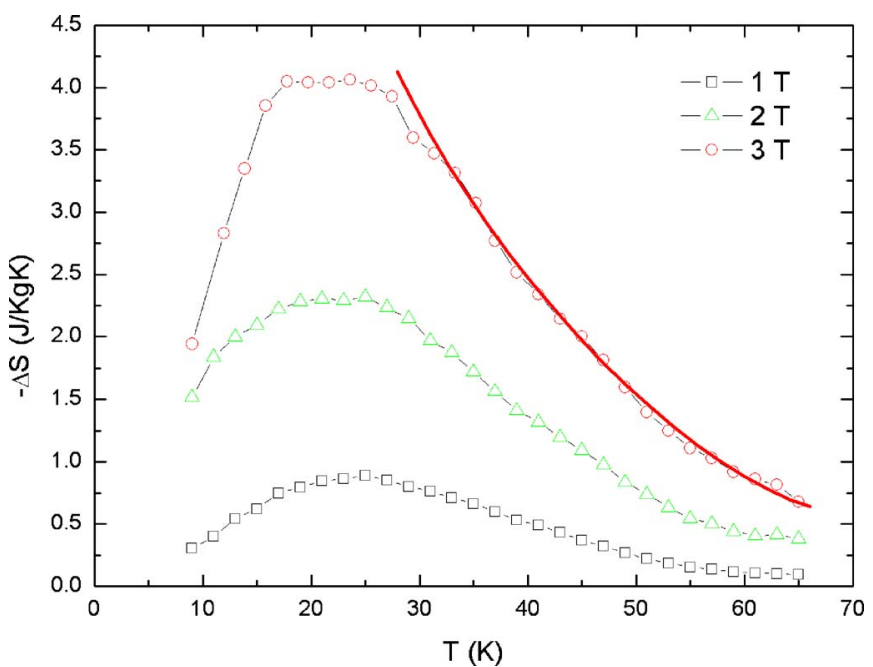

FIG. 7. (Color online) Magnetic entropy change determined from isotherms for the $\mathrm{Tb}_{0.9} \mathrm{Sn}_{0.1} \mathrm{MnO}_{3}$ compound at various magnetic fields. The solid line represents a scaling estimated for higher temperatures from Eq. (4).

reconcile the apparently different evolutions of the maximum (maxima) toward a lower or higher temperature region when the magnetic field increases for various manganite families.

The large magnetocaloric effect in perovskite manganites could originate from the spin-lattice coupling in the magnetic ordering process. ${ }^{20,21}$ The strong coupling between spin and lattice has been shown by the observed lattice changes accompanying magnetic transitions in these manganites. ${ }^{22}$ The lattice structure changes in the $\langle\mathrm{Mn}-\mathrm{O}\rangle$ bond distance as well as in the $\langle\mathrm{Mn}-\mathrm{O}-\mathrm{Mn}\rangle$ bond angle, would in turn favor the spin ordering. Then, a more abrupt variation of magnetization near the magnetic transition occurs and results in a large magnetic entropy change.

The customary estimation of the magnetocaloric materials is based on a comparison of the maximum magnetic entropy change at a magnetic field of $1 \mathrm{~T}$. Following this procedure, one can see that $\Delta S_{M}(T)=-1 \mathrm{~J} / \mathrm{Kg} \mathrm{K}$ of the sample studied is twice as small as compared to the large values reported for $\mathrm{La}_{1-x} \mathrm{Sr}_{x} \mathrm{MnO}_{3}$ in Ref. 18 and other manganites at higher temperatures. ${ }^{6-9}$ In order to evaluate the magnetic cooling efficiency one may apply the so-called relative cooling power $[\mathrm{RCP}(\mathrm{s})]$, which takes into account the width of a temperature interval. ${ }^{19}$ Due to a very sparse study of a magnetocaloric effect below $100 \mathrm{~K}$, the $\mathrm{RCP}(\mathrm{s})$ value being about $55 \mathrm{~J} / \mathrm{kg}$ for a sample studied, may be compared with 55-65 J/kg at 42 and $35 \mathrm{~K}$ as reported for $\mathrm{La}_{0.65} \mathrm{Ca}_{0.35} \mathrm{Ti}_{0.4} \mathrm{MnO}_{3} \quad$ and $\mathrm{La}_{0.83} \mathrm{Ca}_{0.17} \mathrm{Ti}_{0.4} \mathrm{MnO}_{3}$, respectively. ${ }^{19}$

The experimental data for the case of $3 \mathrm{~T}$ and for temperatures above $30 \mathrm{~K}$ could be scaled as follows:

$$
\Delta S_{M}(T) \sim A /\left(T-T_{C}\right)^{\alpha} \quad \text { for } T>T_{C}
$$

where the coefficients were found as $A=61.1 \mathrm{~J} / \mathrm{Kg}, T_{C}$ $=21 \mathrm{~K}$, and $\alpha=1.12$. The scaling is plotted in the figure by the solid line. 


\section{CONCLUSION}

In summary, we have studied the magnetic properties and the magnetocaloric effect of a manganite $\mathrm{Tb}_{0.9} \mathrm{Sn}_{0.1} \mathrm{MnO}_{3}$ polycrystalline sample. The material is paramagnetic above $37 \mathrm{~K}$. For lower temperatures we observe the competition between antiferromagnetic and ferromagnetic interactions. The magnetic transition occurs in temperatures lower than that observed in the pure $\mathrm{TbMnO}_{3}$ compound as a consequence of Sn doping. This kind of substitution partially breaks the magnetic network among the magnetic atoms with the consequent modifications on the magnetic-phase diagram of this system. We have obtained a relatively high value of magnetic entropy change associated with the magnetic transition of this manganite. This relatively large value and broad temperature interval (about $35 \mathrm{~K}$ ) of magnetocaloric effect make the present compound a promising candidate for magnetic refrigerators at low temperatures, where the heat capacities are remarkably reduced.

\section{ACKNOWLEDGMENTS}

This work was supported in part by CGRI (B), Ministry of Science and Higher Education (PL*), grant Walonia/286/ 2006), *Kasa Mianowskiego (PL), and FNRS (B). F.W.F. was a recipient of a postdoc through FRFC, http://1.5.115.3/, 1.5.115.03 convention.

${ }^{1}$ E. Werbung, Ann. Phys. Chem. 13, 141 (1881).

${ }^{2}$ T. Kimura, T. Goto, H. Shintani, K. Ishizaka, T. Arima, and Y. Tokura Nature 426, 55 (2003).

${ }^{3}$ T. Kimura, T. Goto, Y. Tokura, and A. P. Ramirez, Phys. Rev. B 71,
224425 (2005).

${ }^{4}$ M. Kenzelmann, A. B. Harris, S. Jonas, C. Broholm, J. Schefer, S. B. Kim, C. L. Zhang, S.-W. Cheong, O. P. Vajk, and J. W. Lynn, Phys. Rev. Lett. 95, 087206 (2005).

${ }^{5}$ T. Arima, T. Goto, Y. Yamasaki, S. Miyasaka, K. Ishii, M. Tsubota, T. Inami, Y. Murakami, and Y. Tokura, Phys. Rev. B 72, 100102 (2005).

${ }^{6}$ M.-H. Phan, S. C. Yu, and N. H. Hur, J. Magn. Magn. Mater. 262, 407 (2003).

${ }^{7}$ H. Gencer, S. Atalay, H. I. Adiguzel, and V. S. Kolat, Physica B (Amsterdam) 357, 326 (2005)

${ }^{8}$ N. Chau, N. D. Tho, N. H. Luong, B. H. Giang, and B. T. Cong, J. Magn. Magn. Mater. 303, e402 (2006).

${ }^{9}$ L. Morales, R. Zysler, and A. Caneiro, Physica B (Amsterdam) 320, 100 (2002).

${ }^{10}$ Y. Cui, L. Zhang, G. Xie, and R. Wang, Solid State Commun. 138, 481 (2006).

${ }^{11}$ H. Y. Hwang, S.-W. Cheong, P. G. Radaelli, M. Marezio, and B. Batlogg, Phys. Rev. Lett. 75, 914 (1995).

${ }^{12}$ J. M. D. Coey, M. Viret, and S. von Molnar, Adv. Phys. 48, 167 (1999).

${ }^{13}$ E. F. Bertaut and M. Mercier, Phys. Lett. 5, 27 (1963).

${ }^{14}$ M. Bieringer and J. E. Greedan, Solid State Chem. 143, 132 (1999).

${ }^{15}$ A. Munoz, J. A. Alonso, M. J. Martinez-Lope, M. T. Casais, J. L. Martinez, and M. T. Fernadez-Diaz, Phys. Rev. B 62, 9498 (2000).

${ }^{16}$ S. K. Banerjee, Phys. Lett. 12, 16 (1964).

${ }^{17}$ S. Chaudhary, V. Sunil Kumar, S. B. Roy, P. Chaddah, S. R. Krishnakumar, V. G. Sathe, A. Kumar, and D. D. Sarma, J. Magn. Magn. Mater. 202, 47 (1999).

${ }^{18}$ A. Szewczyk, H. Szymczak, A. Wisniewski, K. Piotrowski, R. Kartaszynski, B. Dabrowski, S. Kolesnik, and Z. Bukowski, Appl. Phys. Lett. 77, 1026 (2000).

${ }^{19}$ M. H. Phan and S.-C. Yu, J. Magn. Magn. Mater. 308, 325 (2007).

${ }^{20}$ M.-H. Phan, S.-C. Yu, N. H. Hur, and Y.-H. Yeong, J. Appl. Phys. 96, 1154 (2004).

${ }^{21}$ Z. B. Guo, Y. W. Du, J. S. Zhu, H. Huang, W. P. Ding, and D. Feng, Phys. Rev. Lett. 78, 1142 (1997).

${ }^{22}$ P. G. Radaelli, D. E. Cox, M. Marezio, S.-W. Cheong, P. E. Schiffer, and A. P. Ramirez, Phys. Rev. Lett. 75, 4488 (1995). 